\title{
Flujos de plasma hidrodinámicos y magnetohidrodinámicos. Parte II
}

\section{Hydrodynamic and magnetohydrodynamic plasma flows. Part II}

Presentación: 26/07/2021

Aprobación: 09/09/2021

\section{Néstor Rotstein}

Estudio de energías por fusión de plasmas magnetizados, Facultad Regional Buenos Aires, Universidad Tecnológica Nacional - Argentina

nrotstein@frba.utn.edu.ar

\section{Ignacio Bustelo Cancela}

Estudio de energías por fusión de plasmas magnetizados, Facultad Regional Buenos Aires, Medrano 951 (CP1179) - Buenos Aires, Universidad Tecnológica Nacional - Argentina ignaciobustelo@gmail.com

\section{Resumen}

En este artículo consideramos un flujo de plasma embebido en un campo magnético y confinado por él, y discutimos los valores mínimos (o umbral) del campo magnético que serían necesarios para gobernar la dinámica del flujo. Asimismo introducimos una fuerza estadística desarrollada en base a la teoría de longitudes características de mezcla (mixing length) para modelizar la transferencia de momento mediante una suerte de viscosidad turbulenta originada en procesos turbulentos promediados, con y sin campo magnético. De esta manera extendemos la primera parte de este trabajo, sumando efectos magnéticos y turbulentos a la acción de la fuerza debida al gradiente de presión y a la compresión gravitatoria.

Palabras clave: plasmas - magnetohidrodinámico - turbulencia - energía

\begin{abstract}
In this article we consider a plasma outflow confined by a magnetic field, and we discuss the minimum (or threshold) values of the magnetic field that would be necessary to govern the dynamics of the flow. We also introduce a statistical force developed on the basis of the theory of characteristic mixing lengths to model the momentum transfer through a kind of turbulent viscosity originated in averaged turbulent processes, with and without a magnetic field. In this way we extend the first part of this work, adding magnetic and turbulent effects to the action of the force due to the pressure gradient and gravitational compression.
\end{abstract}

Keywords: plasmas - magnetohydrodynamic - turbulence - energy 


\section{Introducción}

Los fenómenos de eyección de masa son comunes a todos los objetos estelares a lo largo de toda la secuencia evolutiva y engloban un conjunto de mecanismos de intercambio de masa y energía necesarios para conservar el equilibrio de esos objetos con el entorno. Algunos de esos procesos son eventuales, como por ejemplo los procesos disruptivos de las protuberancias solares, y otros continuos, asociados a la expansión permanente de la envoltura y que se conocen con el nombre genérico de vientos.

La interacción de los vientos con los campos magnéticos de los objetos de los que emergen es objeto de estudio desde hace décadas (véanse, por ejemplo, las revisiones de Hollweg, 1978; Kudritzki y Puls, 2000; Owocki, 2013: Shivamoggi, 2020). Entre tanto, las mejoras en instrumentos y métodos ha llevado a la medición de campos magnéticos estelares de muy baja intensidad, incluso de alrededor de $1 G$ (véase Lingnières et al, 2009). Sin embargo, una serie de estudios de campos magnéticos estelares arrojó resultados nulos (Schöller et al, 2011), lo que llevó a una de las preguntas que formulamos en la parte I, referida a que si existe un valor umbral de detección de campo magnético, ¿existe también un valor crítico de ese campo, sólo por encima del cual le es posible gobernar la dinámica del viento?.

El campo magnético (asociado a la actividad interna de una estrella) no sólo confina al plasma a partir de su interacción con el campo de velocidades (y de esta interacción resulta que en el caso de plasma idealmente conductor la geometría del flujo es la del campo magnético) sino que además sustenta en parte al plasma en contra de la compresión gravitatoria sin modificar la densidad. Esto significa que cualquier elemento sometido a presión magnética que esté en equilibrio de presión con su entorno debe tener una temperatura más baja para tener la misma densidad y evitar la flotación dentro del fluido. El ejemplo más cercano que tenemos de confinamiento magnético de un plasma es el que se realiza en los reactores de fusión.

Debemos tener en cuenta además que en el escenario general de un campo magnético confinando un flujo saliente de plasma y el plasma estirando las líneas de campo magnético hasta eventualmente romperlas es posible que se den fenómenos de turbulencia.

Precisamente, en esta parte del trabajo estudiaremos los efectos combinados de campos magnéticos y fuerzas de volumen que asociaremos fenomenológicamente a fuerzas de tipo turbulento. Para ello, en el apartado 2 plantearemos las ecuaciones generales y las resolveremos en la sección 3, dejando para la sección 4 el análisis y discusión de los resultados.

\section{Tratamiento general}

Las ecuaciones magnetohidrodinámicas que gobiernan la evolución estacionaria de un plasma no ideal, interactuando con campos magnéticos externos y fuerzas de volumen en presencia de un campo gravitatorio, se escriben como

$$
\begin{aligned}
& \nabla \cdot \boldsymbol{B}=0 \\
& \nabla \cdot \rho \boldsymbol{v}=0 \\
& \nabla \times(\boldsymbol{v} \times \boldsymbol{B}-\eta \boldsymbol{j})=0 \\
& \rho(\boldsymbol{v} \cdot \nabla) \boldsymbol{v}=-\nabla \mathcal{P}+\frac{1}{\mu_{0}}(\nabla \times \boldsymbol{B}) \times \boldsymbol{B}-\rho g \boldsymbol{e}_{r}+\mathcal{F}
\end{aligned}
$$




$$
(v \cdot \nabla)\left(\frac{\mathcal{P}}{(\gamma-1) \rho}\right)+\mathcal{P}(v \cdot \nabla)\left(\frac{1}{\rho}\right)=\mathcal{Q}(r, \theta)
$$

donde, como es habitual, $\rho$ representa la densidad volumétrica de masa, $v$ el campo de velocidades, $\boldsymbol{B}$ el campo de inducción magnética, gla aceleración gravitatoria, $\mathcal{P}=k_{B} \rho T / \mu m$ (donde $\mu$ es el peso molecular medio, $k_{B}$ la constante de Boltzmann) la presión del fluido y $\eta$ $=1 / \mu_{0} \sigma$ la difusividad magnética. Hemos cerrado las ecuaciones (1)-(4) con la ecuación (5) de balance termodinámico, que representa la distribución de fuentes de calor que de manera consistente completa el sistema de ecuaciones MHD, en la que Q representa la tasa de intercambio de potencia por unidad de masa. Hemos incluido además una fuerza $\mathcal{F}$ para dar cuenta de eventuales intercambios de cantidad de movimiento y/o de energía a lo largo del viento.

Para un plasma idealmente conductor $(\eta=0)$ y sin otras fuerzas que la gravitatoria y la magnética, el modelo está completamente desarrollado en Low y Tsinganos (1987) para curvatura nula del campo magnético, y Rotstein y Ferro Fontán (1995) para campos curvados colimados hacia el polo. A continuación resolveremos el problema para campos curvados hacia el ecuador. Para ello, notemos que todo flujo axisimétrico representado por las ecuaciones (1) y (2), en coordenadas esféricas pueden escribirse en las formas

$$
\begin{aligned}
& \boldsymbol{B}=\frac{1}{r \operatorname{sen} \theta} \nabla A(r, \theta) \times \hat{e}_{\varnothing}+B_{\varnothing} \hat{e}_{\varnothing} \\
& \rho \boldsymbol{v}=\frac{1}{r \operatorname{sen} \theta} \nabla \psi(r, \theta) \times \hat{e}_{\varnothing}+\rho v_{\varnothing} \hat{e}_{\varnothing}
\end{aligned}
$$

donde hemos introducido la función de flujo magnético $A(r, \theta)$ que gobierna la existencia de superficies de flujo magnético, y la función de flujo másico $\psi(r, \theta)$ con un significado funcional similar para el flujo de masa. Ambas funciones de flujo son constantes sobre cada superficie de flujo y describen el flujo poloidal entre las diferentes isosuperficies, con el adicional de que con resistividad nula, y a menos de una constante rotacional, las isosuperficies de flujo másico coinciden con las isosuperficies de flujo magnético. Esto significa que sobre toda y cualquier isosuperficie debe cumplirse

$$
\rho \boldsymbol{v}=\beta \boldsymbol{B}
$$

donde $\beta$ es una constante. Lo que implica esta última identidad es que el flujo magnético $\varphi_{\mathrm{M}}=\iint B \cdot d \boldsymbol{S}$ a través de una superficie $\boldsymbol{S}$ arrastrada por el campo de velocidades es independiente del tiempo. O sea, cualquier tubo de flujo conserva su estructura en tanto se mueve con el flujo (pero esto sólo ocurre en el tratamiento MHD ideal, las líneas de campo se congelan al fluido sólo si la resistividad del plasma es nula). Como en este caso el plasma está congelado al campo magnético, prescribiremos aquí la topología del campo magnético de idéntica manera a como lo hicimos en la parte I de este trabajo para el campo de velocidades, esto es, definiremos la función de flujo magnético como

$$
A(r, \theta)=W(r) \operatorname{sen}^{2} \theta
$$


de donde resultan

$$
\begin{aligned}
& B_{r}=\frac{\partial_{\theta} A}{r^{2} \operatorname{sen} \theta}=\frac{2 W}{r^{2}} \cos \theta \\
& B_{\theta}=-\frac{\partial_{r} A}{r \operatorname{sen} \theta}=-\frac{\partial_{r} W}{r} \operatorname{sen} \theta
\end{aligned}
$$

Habremos de simular la geometría del campo magnético (líneas parcialmente abiertas en las regiones polares y cerradas en la zona ecuatorial) como en la parte I a través de una función general de la forma

$$
W(r, \theta)=k r^{q} \operatorname{sen}^{2} \theta
$$

donde q es el coeficiente que define la curvatura del campo y su región cerrada. Obsérvese que para evitar que el campo diverja, debe ser $q<2$. En la figura 1 mostramos las líneas de campo magnético (que son los lugares del plano poloidal en los que $B(x) \operatorname{sen}^{2} \theta=m=$ constante) para $q=-0.2$ y $q=-1$.
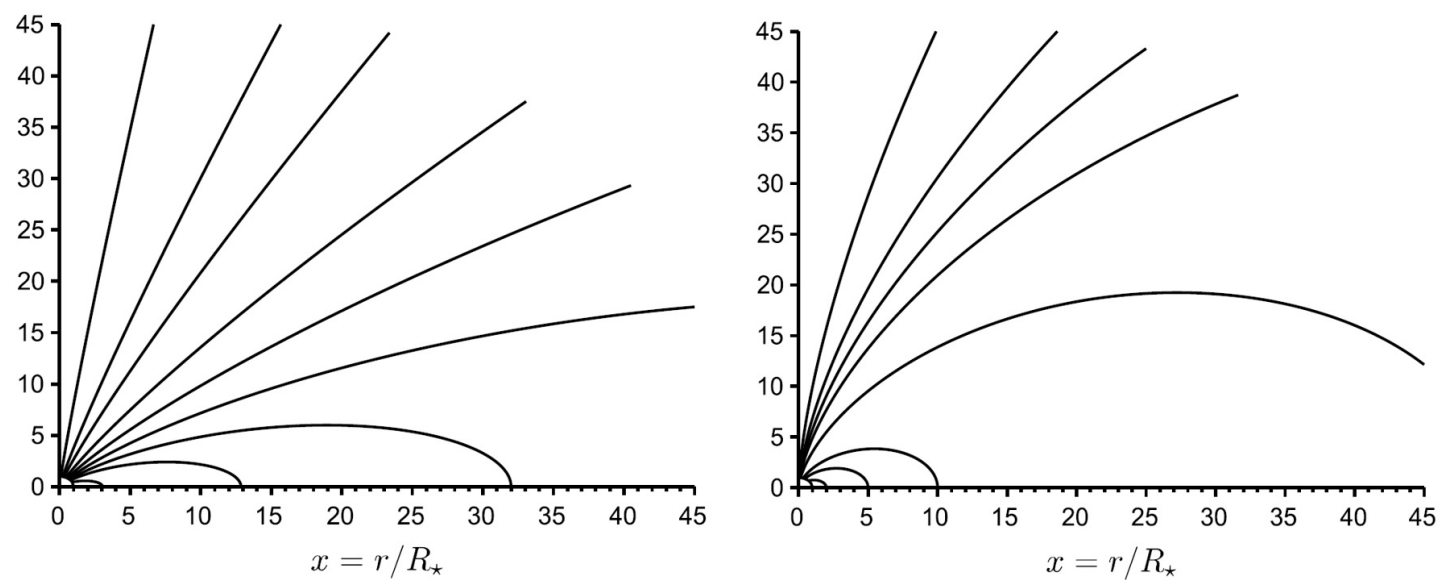

Figura 1: líneas de campo magnético para $q=-0,2$ (panel izquierdo) y $q=-1$ (panel derecho). En ambos casos las líneas son cerradas, pero para $\mathrm{q}=-0,2$ la curvatura de las líneas polares comienza a evidenciarse a alrededor de $200 \mathrm{R}_{\star \prime}$ una región en la que el viento se ha disipado. La diferencia con la gráfica similar de la parte I es que ahora no fijamos directamente las líneas de flujo sino la geometría del campo magnético.

Las distancias las mediremos en términos del parámetro adimensional $x$, que definiremos en términos del radio estelar como

$$
x=\frac{r}{R_{*}}
$$

de manera tal que, teniendo en cuenta que $\partial_{r}=\partial_{x} / R_{\star}$, podamos redefinir las componentes poloidales del flujo como 


$$
\begin{aligned}
& \rho v_{r}=2 \frac{\beta k}{R_{\star}^{2-q}} \frac{1}{x^{2-q}} \cos \theta \equiv \frac{2 \beta \kappa}{x^{2-q}} \cos \theta \\
& \rho v_{\theta}=-q \frac{\beta k}{R_{\star}^{2-q}} x^{q-2} \operatorname{sen} \theta \equiv-q \frac{\beta \kappa}{x^{2-q}} \operatorname{sen} \theta
\end{aligned}
$$

Obsérvese que el significado de la constante $k$ es diferente al de la parte I. De hecho tienen unidades diferentes, en la primera parte de este trabajo representa la densidad del flujo de masa y en este caso es un campo magnético.

Supongamos ahora que la fuerza $\mathcal{F}$ se halla ligada a turbulencia. El origen de la turbulencia está asociado a inestabilidades que se generan por efecto de gradientes de velocidades, que a su vez provocan inestabilidades de escala espacial cada vez menor hasta que eventualmente son lo suficientemente pequeños como para que la viscosidad se torne relevante y la energía mecánica se disipe en forma de calor. De hecho, la turbulencia es un mecanismo eficiente de mezcla de masa y momento, y un mecanismo eficaz de intercambio de energía que se disipa viscosamente a la escala de los torbellinos más pequeños.

Podemos introducir una idea general del problema a primer orden si pensamos en una escala media de los torbellinos, $\ell_{T}$, y reescalamos los gradientes a las dos dimensiones asociadas al plano poloidal. El esquema general es el que presentamos en la figura 2, en la que $\ell_{T}$ representa la longitud de la trayectoria que recorre un elemento de fluido en su movimiento apartado del equilibrio, $\mathrm{y} \ell_{r} \mathrm{y} \ell_{\theta}$ sus proyecciones sobre las direcciones poloidales.

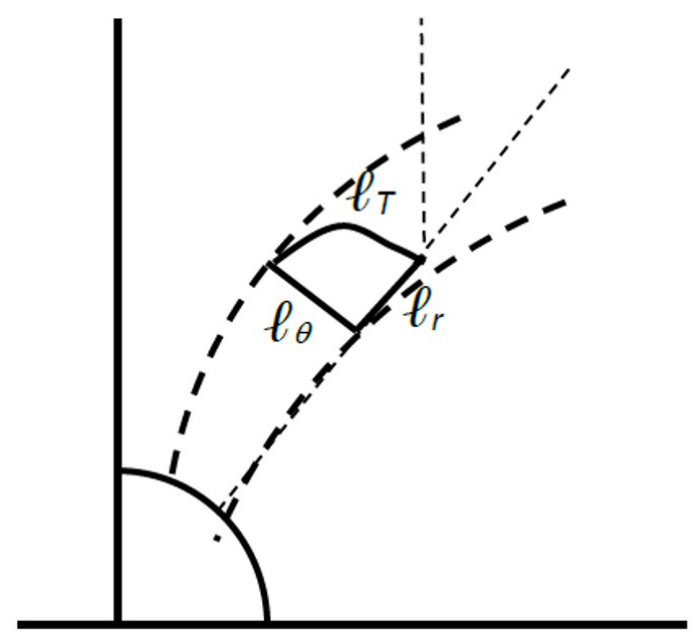

Figura 2: en el esquema se muestran las distancias características que se utilizan para evaluar la fuerza $\mathcal{F} . \ell_{\theta}$ es una distancia en la dirección polar, en tanto que $\ell_{r}$ es la distancia que un elemento de fluido recorre radialmente mientras se desplaza en dirección polar. $\ell_{T}$ es la distancia media turbulenta.

Definamos el tiempo de deriva como aquel que le lleva a un elemento de fluido desplazarse una cierta distancia perpendicular a su trayectoria. Luego, podemos definir los tiempos de deriva como $\tau_{\theta r}=\ell_{\theta} / v_{r}$ y $\tau_{r \theta}=\ell / v_{\theta}$. Ahora bien, la viscosidad cinemática turbulenta, $v$, 
puede pensarse como el producto de una distancia típica de turbulencia, en este caso $\ell_{T}$, por una velocidad turbulenta media característica, que en virtud de la pequeña curvatura del campo, en las regiones del viento, asociaremos a la velocidad radial. Esta velocidad turbulenta media, en consecuencia, queda definida por la distancia radial turbulenta dividida por la velocidad de deriva correspondiente, es decir

$$
v=\ell_{T}\left\langle v_{T}\right\rangle \sim \ell_{T}\left(\ell_{r} \frac{v_{r}}{\ell_{\theta}}\right) \sim \ell_{T}\left(v_{r} \operatorname{tg} \theta\right) \sim \ell_{T} \partial_{\theta} v_{r}
$$

A su vez, habremos de asociar las componentes de la fuerza $\mathcal{F}$ a los gradientes laterales de las componentes propias de velocidad, es decir, definiremos estas componentes en la forma

$$
\begin{aligned}
& \mathcal{F}_{r}=\frac{\lambda}{\ell_{T}} \rho v\left(\ell_{r} \frac{v_{r}}{\ell_{\theta}}\right)=\frac{\lambda}{\ell_{T}} \rho \ell_{T} \partial_{\theta} v_{r}\left(\ell_{r} \frac{v_{r}}{\ell_{\theta}}\right)=\frac{4 \lambda}{R_{\star} \mathcal{L}} \frac{H}{x^{2-q}} \operatorname{sen}^{2} \theta \\
& \mathcal{F}_{\theta}=\frac{\lambda}{\ell_{T}} \rho v\left(\ell_{\theta} \frac{v_{\theta}}{\ell_{r}}\right)=2 \frac{\lambda q}{R_{\star} \mathcal{L}} \frac{H}{x^{2-q}} \operatorname{sen} \theta \cos \theta
\end{aligned}
$$

donde $\lambda$ es un parámetro adimensional (cuyo valor es $0,+1$ ó -1 ) y $\mathcal{L}=\ell, R_{\star}$ es el tamaño adimensionalizado típico de un torbellino. Además, hemos definido la función $H(x)$ en la forma

$$
H=\frac{\beta^{2} \kappa^{2}}{\rho x^{2-q}}
$$

Obsérvese que la función $H(x)$ tiene unidades de presión, de manera tal que definiremos su forma adimensional en términos de la presión magnética en la base del viento como

$$
\mathcal{H}(x)=\frac{H}{P_{M}}=\frac{H}{B^{2} / 2 \mu_{0}}
$$

donde $B^{2}=B_{r \star}^{2}+B_{\theta \star}^{2}=\left(4+q^{2}\right) \kappa^{2}$ Como en la parte $\mathbf{I}$, tomaremos la presión en la forma

$$
\mathcal{P}(r, \theta)=P_{1}(r)+P_{2}(r) \operatorname{sen}^{2} \theta
$$

de manera tal que, con velocidad azimutal nula y simetría de rotación, las componentes poloidales de la ecuación (4) resultan entonces

$$
4 \frac{\partial_{x} \mathcal{H}}{x^{2-q}}=-\partial_{x}\left(\frac{P_{1}}{P_{M}}\right)-\frac{P_{G R}}{P_{M}} \frac{\rho}{\rho_{\star} x^{2}}
$$




$$
\begin{aligned}
& -4 \frac{\partial_{x} \mathcal{H}}{x^{2-q}}+q(2-q) \frac{\mathcal{H}}{x^{3-q}}=-\partial_{x}\left(\frac{P_{2}}{P_{M}}\right)-2 \frac{\kappa^{2}}{B^{2}} \frac{q[q(q-1)-2]}{x^{5-2 q}}+\frac{4 \lambda}{\mathcal{L}} \frac{\mathcal{H}}{x^{2-q}} \\
& -q \frac{\partial_{x} \mathcal{H}}{x^{1-q}}-q\left(1-\frac{q}{2}\right) \frac{\mathcal{H}}{x^{2-q}}=-\left(\frac{P_{2}}{P_{M}}\right)-2 \frac{\kappa^{2}}{B^{2}} \frac{[q(q-1)-2]}{x^{4-2 q}}+\frac{\lambda q}{\mathcal{L}} \frac{\mathcal{H}}{x^{1-q}}
\end{aligned}
$$

donde, como en la parte I, hemos definido $P_{G R}=\left(\rho_{*} G M_{*}\right) / R_{*}$, y $\rho_{*}$ como la presión gravitatoria y la densidad en la base del viento $\left(r=R_{\star} \equiv x=1\right)$, respectivamente.

\section{Soluciones MHD}

En el caso de rotación nula $\left(v_{\varphi^{*}}=0\right)$, derivando respecto a $x$ la expresión (24), e igualando a la ecuación (23), obtenemos la ecuación para la variable $\mathcal{H}$ en la forma

$$
\begin{gathered}
-q \frac{\partial_{x}^{2} \mathcal{H}}{x^{1-q}}+\left(4-\frac{q^{2}}{2}+x \frac{\lambda q}{\mathcal{L}}\right) \frac{\partial_{x} \mathcal{H}}{x^{2-q}}-\left(\frac{q^{2}}{2}(2-q)+x \frac{\lambda}{\mathcal{L}}(4+q(1-q))\right) \frac{\mathcal{H}}{x^{3-q}}= \\
=2 \frac{\kappa^{2}}{B^{2}} \frac{(4-q)[q(q-1)-2]}{x^{5-2 q}}
\end{gathered}
$$

Es casi evidente que la fuerza de Lorentz $\boldsymbol{j} \times \boldsymbol{B}$ forma parte sólo de la solución particular, pero eso no significa que este caso pueda reducirse al hidrodinámico con sólo anular el miembro derecho, porque dependiendo de las fuerzas involucradas, debemos prestar atención al significado de la función $H(x)$ en cada caso. Por ejemplo, para un flujo hidrodinámico representa una velocidad, en tanto que en este caso representa una presión. Téngase en cuenta, además, que con resistividad nula la ecuación (3) indica que el flujo de plasma y el campo magnético evolucionan congelados uno al otro.

En el caso en que no haya otras fuerzas de volumen $(\lambda=0)$, la ecuación de definición es la misma expresión (23) de la parte I, y es fácil ver que la parte magnética sólo interviene como solución particular. Luego, resulta

$$
\mathcal{H}(x)=A x^{s 1}+B x^{s 2}+\frac{2}{4+q^{2}} x^{q-2}
$$

donde, para el valor de curvatura que estamos tomando ( $q=-0.2$, como en la parte I) resultan $s_{1} \approx 0.01 \quad s_{2} \approx-20$. Debemos recalcar que la diferencia entre la solución (26) y la que obtuvimos en la parte I radica no sólo en el agregado del tercer sumando del miembro derecho, sino en la interpretación de la función $H(x)$.

En caso de incluir las fuerzas de volumen, debemos notar que la fuerza $\mathrm{F}$ crece con la disminución de la escala $\mathcal{L}$ (véanse las ecuaciones (17) y (18)). Por otro lado, debe notarse que estas fuerzas de volumen no desaparecen para un campo potencial de curvatura nula $(q=0)$, en cuyo caso la ecuación (25) se reduce a la forma 


$$
4 \frac{\partial_{x} \mathcal{H}}{x^{2}}-4 \frac{\lambda}{\mathcal{L}} x \frac{\mathcal{H}}{x^{3}}=-16 \frac{\kappa^{2}}{B^{2} x^{5}}=\frac{4}{x^{5}}
$$

y cuya solución resulta

$$
\mathcal{H}(x)=C_{1} e^{x / \mathcal{L}}+\frac{1}{2 x^{2}}-\left(\frac{\lambda}{\mathcal{L}}\right) \frac{1}{2 x}+\frac{1}{2}\left(\frac{\lambda}{\mathcal{L}}\right)^{2}\left[e^{\lambda x / \mathcal{L}} \ln (x)+e^{\lambda x / \mathcal{L}} \sum_{n \geq 1}\left(\frac{\lambda x}{\mathcal{L}}\right)^{n} \frac{1}{n ! n}\right]
$$

Lo primero que puede notarse es que soluciones físicamente aceptables existen sólo para $\lambda<0$, pues en caso contrario la función $\mathrm{H}(\mathrm{x})$ diverge. De esta manera, la fuerza $\mathcal{F}$ es una fuerza de frenado, asociada a escalas de longitud $\mathcal{L}$.

Supongamos ahora $\mathrm{q} \neq 0$. Planteando la solución general como $\mathcal{H}=\sum_{n \geq 0} a_{n} \mathrm{x}^{n+s}$ después de un álgebra convencional la solución homogénea adopta la forma

$$
\mathcal{H}_{h}(x)=A 1 \sum_{n \geq 0}\left(\frac{5 \lambda|q|}{\mathcal{L}}\right)^{n} \frac{1}{\left(1+\frac{n}{18}\right)} \frac{x^{n}}{n !}+\frac{A 2}{x^{20}}\left[1+\frac{2}{19}\left(\frac{5 \lambda|q|}{\mathcal{L}}\right) x+\frac{2}{684}\left(\frac{5 \lambda|q|}{\mathcal{L}}\right)^{2} x^{2}\right]
$$

donde $\mathrm{A} 1$ y A2 son dos constantes en principio arbitrarias. Obsérvese de paso que de manera casi inmediata, en el caso $\lambda=0$ se recupera la función $H(x)$ para los flujos hidrodinámicos que introducimos en la parte I (téngase en cuenta que la parte magnética ingresa como solución particular). A su vez, la solución de la ecuación particular resulta en la forma

$$
\mathcal{H}_{P}=A 3(\alpha) e^{-\alpha x}\left[\ln (x)+\sum_{n \geq 1} \frac{(\alpha x)^{n}}{n ! n}\right]+A 4(\alpha) \quad\left(\alpha=\frac{5 \lambda|q|}{\mathcal{L}}\right)
$$

donde A3( $\alpha$ ) y A4( $\alpha$ ) son dos constantes que dependen del particular valor que adopte el parámetro a. En virtud de la curvatura del campo que estamos tratando, el parámetro a es básicamente la inversa de la razón entre la distancia de disipación y el radio estelar. Para analizar estos resultados regresemos las ecuaciones (14), (19) y (20) de las que puede obtenerse

$$
\mathcal{H}(x)=\frac{v_{r}(x)}{v_{r, 0}} \quad\left(v_{r, 0}=\frac{2 \kappa}{\beta \mu_{0}}\right)
$$

En la figura 3 graficamos la función velocidad radial polar para $\alpha=5\left(\ell_{T}=0,2 \mathrm{R}_{*}\right)$ y para $\alpha=8$ $\left(\ell_{T}=0,125 R_{*}\right)$. Un par de comentarios se hacen necesarios para la interpretación de estas curvas. En primer lugar, $v_{r, 0}$ no representa la velocidad en la base del viento, es sólo un factor de adimensionalización que relaciona parámetros típicos del campo magnético y el campo de velocidad. A su vez, también a partir de las ecuaciones (14), (19) y (20), para un campo magnético de $1 \mathrm{G}$ y una densidad de masa en la base $\rho_{\star}=10^{-\mathrm{m}} \mathrm{kg} / \mathrm{m}^{3}(\mathrm{~m}>0)$ la ecuación (31) en la base adopta el valor 


$$
\mathcal{H}(1)=0.03110^{-m+3} v l\left(v_{r \star}^{2}\right) \equiv 0.031 h
$$

donde $v l\left(v_{r \star}^{2}\right)$ se refiere a la cantidad de velocidad polar radial en la base (esto es, la velocidad en la base sin unidades, aunque expresada en términos del sistema internacional). Obsérvese que $h=1$ sólo habla del valor de la energía cinética del flujo en la base y no de los valores de densidad de masa y de velocidad.
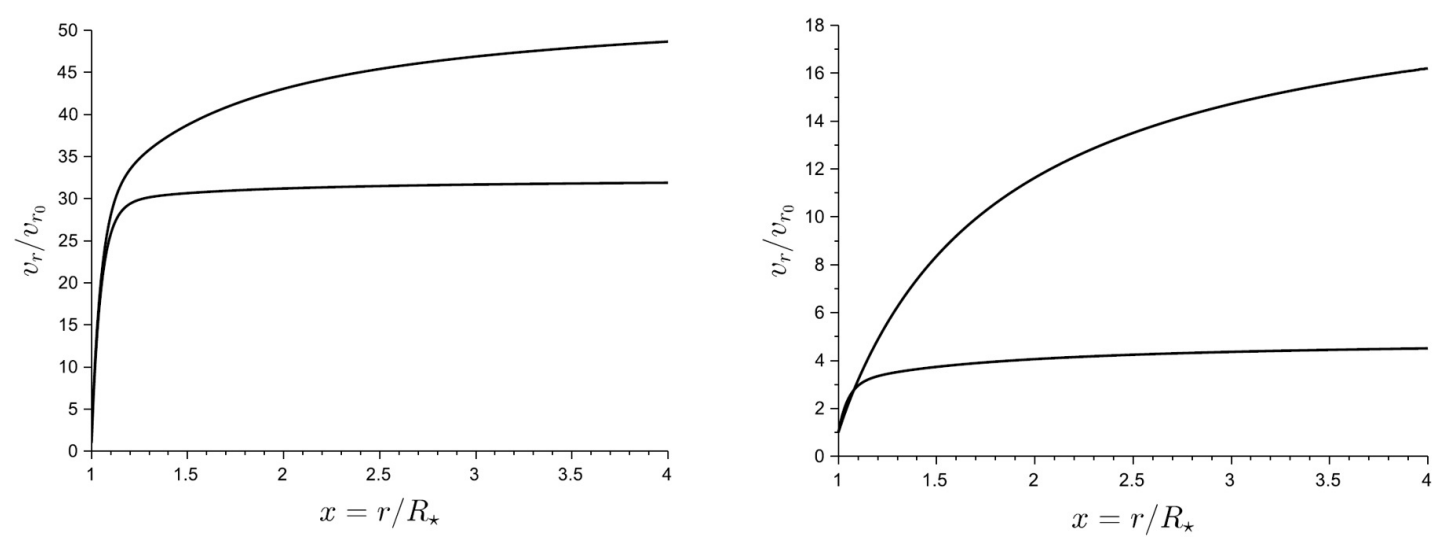

Figura 3: Gráficas de velocidad radial polar para $\alpha=5$ (panel izquierdo) y $\alpha=8$ (panel derecho). En ambos casos la curva superior corresponde a $h=0.1$ y la inferior a $h=1$. Para los valores típicos de densidad $\left(10^{-13} \mathrm{~kg} / \mathrm{m}^{3}<\rho_{*}<10^{-10} \mathrm{~kg} / \mathrm{m}^{3}\right)$ los valores iniciales corresponden a velocidades radiales polares comprendidas en el intervalo $3 \mathrm{~km} / \mathrm{seg} \lesssim v r \lesssim 100 \mathrm{~km} / \mathrm{seg}$. La relación entre parámetros es A1/A2 0,6, salvo para la curva superior del panel derecho, en el que es A1/A2 0,8. Todas las curvas tienden asintóticamente a su valor máximo, a una distancia entre 2 y 10 radios estelares.

Observemos por ejemplo el panel de la izquierda de la figura $3(a=5)$. Dado que la velocidad terminal de estos flujos es del orden de los $400-500 \mathrm{~km} / \mathrm{seg}$, la curva inferior podría corresponder a un flujo inicial del orden de $10 \mathrm{~km} / \mathrm{seg}$ con una densidad de masa correspondiente de alrededor de $10^{-11} \mathrm{~kg} / \mathrm{m}^{3}$. La curva superior, en cambio, podría corresponder a un flujo también con velocidad inicial de $10 \mathrm{~km} / \mathrm{seg}$ pero ahora con una densidad de masa un orden de magnitud inferior.

El escenario del panel derecho $(a=8)$ es diferente. En este caso puede pensarse en flujos del orden de 50 - $100 \mathrm{~km} / \mathrm{seg}$ en la base y densidad del orden $10^{-13}-10^{-14} \mathrm{~kg} / \mathrm{m}^{3}$ para $h=1$.

Por lo pronto, la diferencia esencial entre las curvas se refiere no tanto a la energía cinética en la base sino a la aceleración del flujo. Para $h=1$ y valores comparativamente pequeños de $a(a \lesssim 6)$ esto ocurre en una distancia del orden de $1 / a$, en la que el flujo alcanza alrededor del 98\% de su velocidad terminal. Para valores mayores del parámetro la distancia de régimen terminal crece con a. Obsérvese la figura 4, en la que hemos graficado hasta menos de $1,5 \mathrm{R}_{\star}$ para resaltar esta característica. 


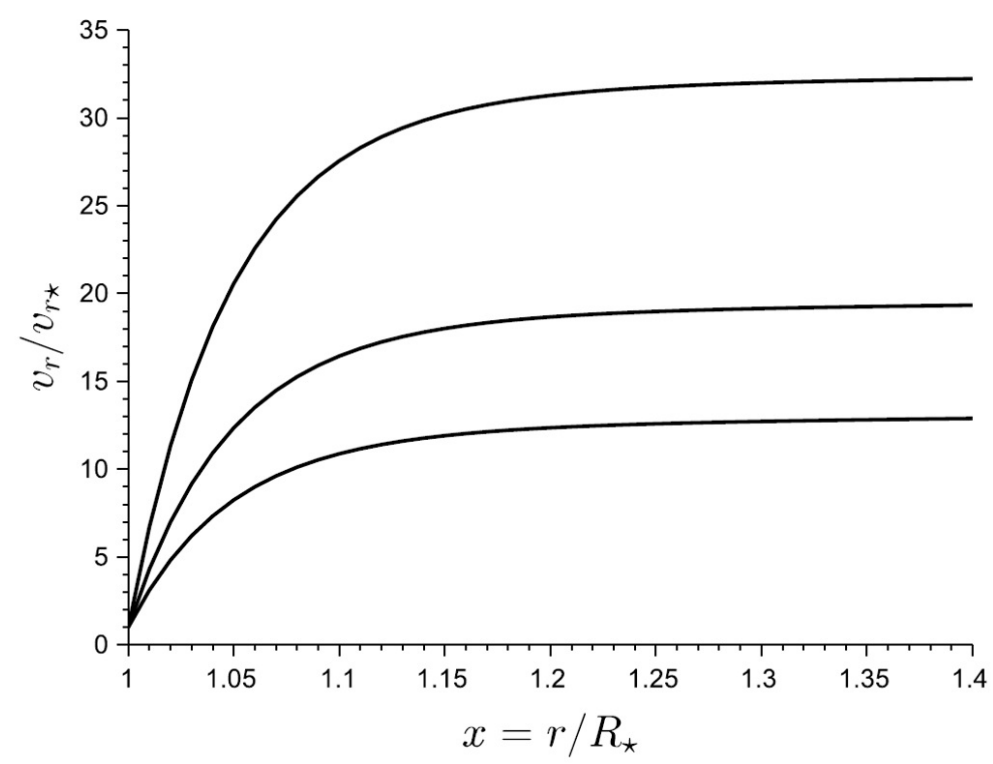

Figura 4: Curvas de velocidad para $\alpha=15, h=1$, en cada uno de los casos para una relación A1/A2 0,6. El $98 \%$ de la velocidad terminal del flujo se da (de arriba hacia abajo) en $x=1,23 ; x=1,24$ y $x=1,26$.

Un aspecto que es necesario remarcar tiene que ver con la intensidad de los campos magnéticos a los que se haya asociado el flujo. Las figuras 3 y 4 representan la velocidad del flujo habiendo asumido un campo magnético de $1 \mathrm{G}$ en la superficie y una relación fija entre las constantes A1 y A2. Todos estos valores pueden ser diferentes aún para una misma curva porque lo único que hemos establecido es una determinada relación entre tres parámetros, a saber, la velocidad inicial, la densidad inicial y la intensidad del campo magnético (o sea, el valor del parámetro $\beta$ ). La velocidad y la densidad se relacionan a su vez a través del parámetro $h$. A modo de ejemplo, regresemos a la curva izquierda inferior, $a=5, h=1$. Como ya hemos dicho, esta curva está construida para un campo $\mathrm{B}_{*}=1 G$. Una posible solución es $v_{*}=10$ $\mathrm{km} / \mathrm{seg}, \rho_{*}=10^{-11} \mathrm{~kg} / \mathrm{m}^{3} \quad\left(\mathrm{n} \sim 10^{20} \mathrm{~cm}^{-3}\right)$. Estos valores son posibles, y claramente estamos en el límite de detección del campo magnético (Lingnières et al, 2009).

Si pensamos en un campo un orden de magnitud mayor, $B_{*}=10 G$, la curva mantiene el perfil y los valores, pero ahora si deseamos mantener la densidad en la superficie debemos asumir una velocidad inicial del orden de $100 \mathrm{~km} / \mathrm{seg}$, de manera tal que la velocidad terminal sería del orden de los $3000 \mathrm{~km} / \mathrm{seg}$, un valor extremadamente alto en estos objetos. Y si la velocidad terminal fuera de, digamos, $500 \mathrm{~km} / \mathrm{seg}$, deberíamos pensar en una densidad del orden $\rho_{*}=2 \times 10^{-6} \mathrm{~kg} / \mathrm{m}^{3}\left(\mathrm{n} \sim 10^{15} \mathrm{~cm}^{-3}\right)$, una densidad excesivamente baja.

Estas propiedades se ven reflejadas inmediatamente en la temperatura del flujo en las regiones de disipación. Obsérvese la figura 5, en la que hemos graficado los perfiles de temperatura en la región del polo, esto es, en la región de máximo flujo.

En ambos casos hemos considerado una densidad media de partículas del orden de $10^{19}-10^{20} / \mathrm{m}^{3}$ (tengamos en cuenta que la fotósfera solar tiene una densidad media de partículas del orden de $10^{21} / \mathrm{m}^{3}$ ). En el caso de la curva inferior de la figura 4, la temperatura en la base del viento es de alrededor de $12.400 \mathrm{~K}$, en tanto que la curva superior se corresponde con una temperatura inicial de alrededor de $11.500 \mathrm{~K}$. En ambos casos se alcanza un pico del orden de $100.000 \mathrm{~K}$. 


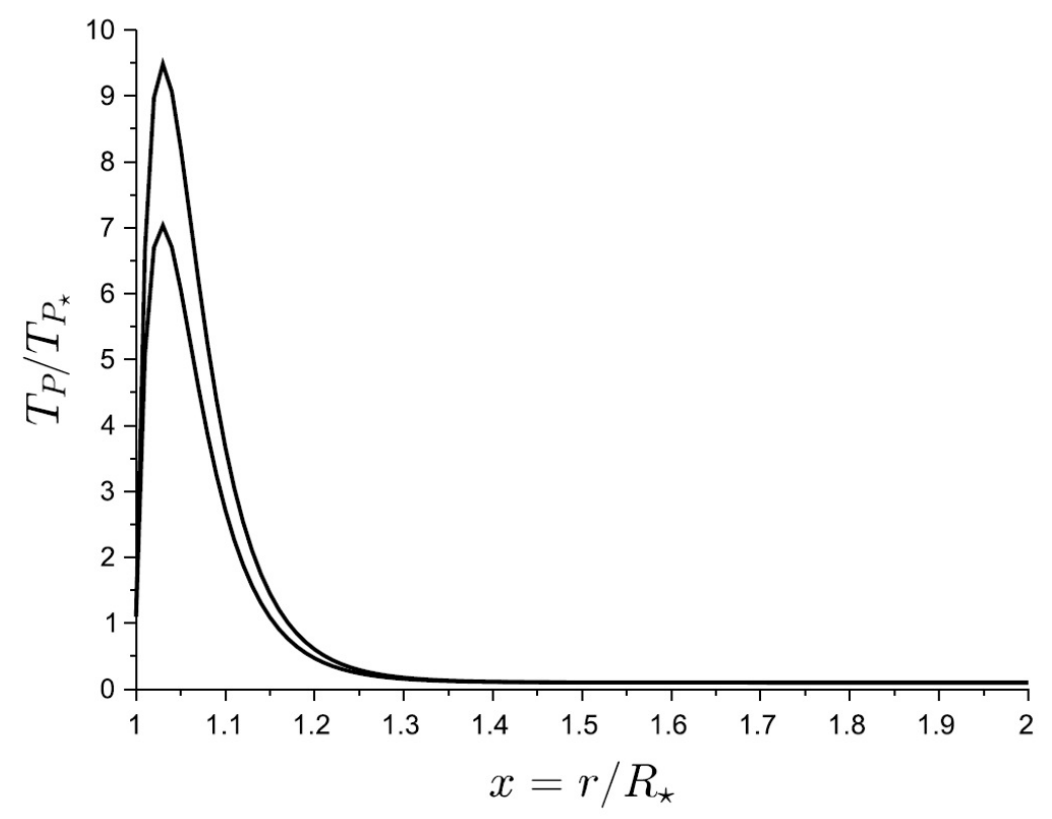

Figura 4: perfiles de temperatura polar para $\square=5, \square=1$ (curva inferior del panel izquierdo de la figura 3) para A1/A2 प0,61 (curva inferior) y A1/A2 प0,59 (curva superior). La temperatura en la base para la curva inferior es del orden de $12.400 \mathrm{~K}$, en tanto que el perfil superior corresponde a una temperatura en la base de alrededor de 11.500K. La velocidad terminal del viento para la curva inferior es de cerca de $300 \mathrm{~km} / \mathrm{seg}$, en tanto que para la otra curva se ubica alrededor de los $430 \mathrm{~km} / \mathrm{seg}$.

Estos valores de temperatura son propios de los sistemas con los que estamos tratando, en los que en lugar de una cromósfera y una corona (como en el caso de las estrellas de tipo solar) la temperatura crece hasta un valor máximo para decrecer luego monótonamente.

Debe notarse, sin embargo, que si bien el perfil se corresponde con datos basados en evidencia observacional, este modelo la temperatura evoluciona en distancias típicamente muy pequeñas, del orden de unas centésimas de radio estelar, un orden de magnitud por debajo de lo esperable. Esto se debe a que no hemos desarrollado un modelo de turbulencia y de disipación de energía turbulenta sino que, en base a criterios de plausibilidad, fijamos una forma de disipar esa energía en escalas que podrían representar las escalas intermedias de los torbellinos. Para decirlo en otras palabras, la figura 4 podría corresponder a perfiles de temperatura en la región de disipación (de hecho, puede verse que decae prácticamente a cero en no más de medio radio estelar) y posiblemente estuviera montada sobre un perfil de comportamiento similar pero que se desarrolla en distancias mayores. Ese análisis escapa por el momento a los objetivos que nos hemos planteado en este trabajo.

Por otro lado debemos tener en cuenta que el hecho de que la velocidad se estabilice rápidamente en su valor asintótico, teniendo en cuenta que el flujo de masa tiende a cero, implica que la densidad de masa decae rápidamente a cero, como puede deducirse de la figura 3 y de la expresión 19. En rigor, existe una rápida descompresión del viento porque la energía interna se deriva en parte a acelerar el flujo y en parte a elevar la temperatura de la envoltura estelar, lo que lleva a su vez a que la densidad de masa decrezca muy rápidamente, como lo hace la presión. 
Sin embargo, la presión, en los límites de la región de disipación de energía, comienza a decrecer más rápidamente que la densidad, lo que provoca el pico en el perfil de velocidad. Pero como el modelo predice una disipación de energía muy rápida, el pico decae también en distancias comparativamente pequeñas.

\section{Comentarios y conclusiones}

En este artículo hemos propuesto un modelo de flujo de plasma magnetizado, congelado al campo magnético externo, en el que se incluyen fuerzas de volumen que de manera fenomenológica hemos asociado a fenómenos turbulentos. Introducidas ad hoc, el desarrollo de las ecuaciones mostró sin embargo que estas fuerzas son naturalmente disipativas, es decir, en ningún momento impusimos el carácter disipativo como condición inicial sino surgió de las soluciones, de ahí que resultara $\lambda=-1$. En principio este resultado era de esperar, porque un modelo en el que este tipo de fuerzas de volumen fueran impulsivas derivaría en velocidades del flujo (intermedias o terminales) que carecerían en principio de evidencia observacional.

En términos de estas fuerzas, hemos propuesto una escala típica de acción. Por su naturaleza disipativa, y asociada a turbulencia, hemos asociado esas escalas con las escalas intermedias de los torbellinos comparativamente más pequeños, aunque en principio un modelo completo de turbulencia hubiera requerido trabajar en toda el dominio de la cascada de energía de Kolmogorov. Nuestra escala $\ell$ es la de disipación de la energía, y en principio el modelo nada dice de que esa disipación sea por efectos de la viscosidad turbulenta. Pero parece ilustrativo sin embargo que al asociar una medida de viscosidad turbulenta el modelo resultara autoconsistente. $Y$ dado que las escalas pequeñas, a diferencia de las grandes, no dependen ni de las condiciones iniciales ni de las condiciones de contorno del sistema, fue posible trabajar de manera aproximada y desligada de las escalas más grandes.

Si aceptamos esta analogía, debemos suponer que nuestra escala de acción de las fuerzas disipativas, $\ell$ ( $\ell_{T}$ en la ecuación de definición (16)), es mucho menor que la longitud de disipación de Kolmogorov, $\ell_{K}$, porque sólo en la suposición $\ell_{T}<<\ell_{K}$ las inestabilidades se tornan irrelevantes y la disipación de energía se produce por efecto de la viscosidad. En este sentido vale la pena mencionar un aspecto relacionado con las curvas de la figuras 3 y 4 . Lo primero que puede notarse es que el régimen de velocidad terminal se alcanza no muy lejos de la superficie, precisamente a distancias comparables con la escala de disipación que impusimos. Este hecho, en principio, estaría asociado a la eficiencia del mecanismo disipativo. Pero por otro lado es notable que al disminuir el valor de la escala típica de disipación turbulenta $\ell_{T}$ la distancia a la que la velocidad alcanza el $98 \%$ de su valor terminal se encuentra algo más lejos de la base. Por ejemplo, para $\alpha=15\left(\ell_{T}=0,066 \mathrm{R}_{*}\right.$ el régimen terminal se alcanza a aproximadamente $0,24 R_{*}$, en tanto que para $\alpha=5$ esto ocurre a no más de $0,15 R_{*}$.

Este fenómeno puede explicarse teniendo en cuenta que la fuerza que introducimos en las expresiones (17) y (18) son de frenado. Al disminuir $\ell_{T}$ aumenta la fuerza $\mathcal{F}$ y en consecuencia la velocidad crece más lentamente. La transferencia de energía desde la base requiere en consecuencia de distancias algo mayores para ser absorbida por el flujo. En este sentido existe cierta familiaridad con los modelos turbulentos. En este tipo de modelos, al disminuir $\ell_{T}$ disminuye el valor de la viscosidad cinemática turbulenta. Para un mismo valor de velocidad turbulenta media y de densidad de masa la tasa de inyección y de disipación de energía habrá de ser similar, pero la distancia de disipación de Kolmogorov va como $v^{3 / 4}$, de manera tal que $\ell_{K}$ disminuye más que $\ell_{T}$, los torbellinos que pueden disipar energía viscosa son más pequeños, y en consecuencia a la distancia típica de disipación todavía 
queda buena parte de la energía inyectada por disipar, provocando en consecuencia que el régimen terminal se logre más alejado de la superficie.

Finalmente, vale la pena mencionar que los valores de temperatura en la base del viento son los que se aceptan para objetos como los que estamos tratando, como lo son las densidades de masa y velocidades. Pero estos valores, al menos para los modelos que hemos desarrollado, tienen sentido en campos magnéticos del orden o menores de 1G. Pero en tal caso o bien estamos en el límite detectable o directamente no se mediría, de manera tal que en principio un campo magnético de relativamente baja intensidad puede influir (e incluso gobernar) en la dinámica de un viento. Y aun cuando las distancias a las que ocurren el pico de temperatura y se alcanza el régimen terminal de flujo son algo menores que las esperables, el modelo tiene también la ventaja de mostrar que a pequeñas escalas los fenómenos que ocurren pueden incidir de manera fundamental en el proceso de eyección de masa. 


\section{Referencias}

Hollweg, J (1978): Reviews of Geophysics, 16, 689. DOI: 10.1029/RGO16i004p00689

Kudritzki, R; Puls, J (2000): Annual Review of Astronomy and Astrophysics, 38, 613, DOI 101146/annurev.astro.38.1.613

Lingnières, F; Petit, P; Böhm, T; Aurière, M (2009): Astron. and Astrophys. Letters, 500, L41, DOI: $10.1051 / 0004-6361$

Low, B; Tsinganos, K (1986): Astrophys. J, 302, 163. DOI 10.1086/163980

Owocki, S (2013): en Planets, Stars and Stellar Systems, Vol. 4, 735. Ed: Oswalt, T.; Barstow, M, ISBN 978-94-007-5614-4. (Springer Science+Business Media, Dordrecht). DOI: 10.1007/978.94.007.5615.1_15

Rotstein, N; Ferro Fontán, C (1995): Astrophys.J, 449, 745 DOI 10.1086/176095

Schöller, M; Hubrig, S; Ilyin, I; Kharchenko, N; Briquet, M; Gonzalez, J; Langer, N; Osnikova, L; the MAGORI Collaboration (2011): Astronomische Nachrichten, 332, 994.

Shivamoggi, B.K (2020): Physics of Plasmas, 27, 012902. DOI: 10.163/1.5127070 